\title{
A Conceptual Design for Reconfigurable Robots
}

\author{
Farhad Aghili \\ Canadian Space Agency * \\ Email: farhad.aghili@space.gc.ca
}

\section{abstract}

The paper presents a new paradigm and conceptual design for reconfigurable robots. Unlike conventional reconfigurable robots, our design doesn't achieve reconfigurability by utilizing modular joints. But the robot is equipped with passive joints, i.e. joints with no actuator or sensor, which permit changing the Denavit-Hartenberg (DV) parameters such as the arm length, and the twist angle. The passive joints are controllable when the robot forms a closed kinematic chain. Also each passive joint is equipped with a builtin brake mechanism which is normally locked but it can be released whenever changing of the parameters is required. Kinematics analysis of such a robot plus control synthesis and mechanical design of the brake mechanism are described.

\section{Introduction}

Robotic manipulators working in extreme environment often need to change their configuration and size in order to meet the environmental and other demands of a given task. Particularly in space application [9], it is desirable and cost effective to employ a single versatile robot capable of performing different tasks such as; inspection, contact operation, assembly (insertion/removal of an object), or carrying an object (pick and place). Optimal operation of each of these tasks demands a specific manipulator design. For instance, large size robots maximizing the structural length index are typically suitable for inspection [1], a robot with the maximum manipulability measure is well-conditioned for a dextrous contact task operation, and a certain configuration maximizing the distance of the robot limbs and extremities from the environment is suitable for payload handling.

The original reconfigurable robot was introduced in $[2,3]$ to add versatility to the robotic manipulator.

\footnotetext{
* (C) Canadian Space Agency 2004
}

This concept was then further developed in [4]. Cellular robots based on hexagonal modules and on robot molecule were described in $[5,6]$ and in $[7,8]$, respectively. Reconfigurable robots for space exploration proposed in [9]. The design of Conro modules to build deplorable modular robots that can reconfigure into different shapes such as snakes or hexapods presented in [10] that has some similarities with Tetrobot [11]. All these reconfigurable robots are modular and hence they need an effective docking system for connector for joining and releasing the modules [12].

In this paper, we describe the conceptual design of a configurable robot that can changes its kinematic design parameters, i.e. the Denavit-Hartenberg (DV) parameters, without requiring modular joint. Such a robot could be used in the aforementioned applications that would benefits from the use of different size and configuration. In this design, the robot have passive joints as many as adjustable DV parameters - prismatic joints and rotary joints correspond to the linear and angular parameters. The passive joints do not have any actuator or senor but they are equipped with a brake or a locking device which is normally locked and can be unlocked by activating a solenoid. In addition to the passive joint, the robot has conventional active joints with actuators and sensors. It is these passive joints which determine the desired kinematic configuration of the manipulator. The process of changing manipulator configuration follows these sequences: (I) the robot manipulator forms a closed chain kinematic by grasping a fix point, (II) all brakes are released and that gives rise to the number of the manipulator's joints, i.e. the active joints together with the passive joints, (III) the desired kinematic configuration is translated to desired passive joints, and the closed chain system is controlled in such a way that the desired joint positions are achieved, (IV) upon converging to the desired joint values, the brakes are locked again and subsequently the manipulator endeffector releases the grasping point. Then, a manipulator with new configuration is born. 

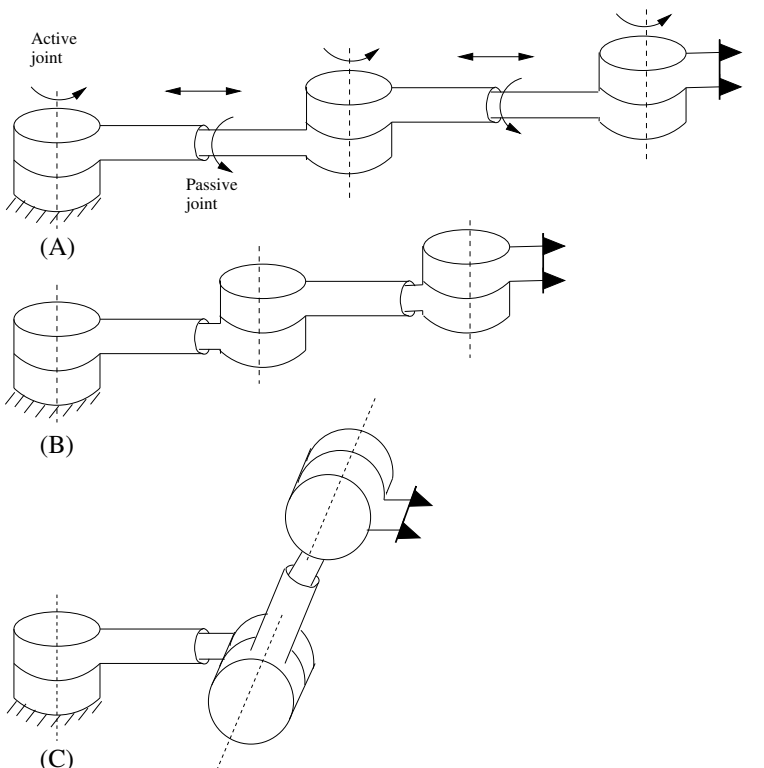

Figure 1: A reconfigurable robot.

This paper is organized as follow. Section 2 summarizes the philosophy behind the new design of the reconfigurable robots. In section 3 , we describe the kinematics analysis of the reconfigurable robots considering the DV parameters as passive joints. Control of the passive joints when the robots is constrained is presented in Section 4. Finally, in Section 5 we describe mechanical design of a built-in brake for locking the passive joints.

\section{Philosophy of Design}

The goal of this project is to build reconfigurable robots with capabilities to change their kinematics parameters according to demands of a task. Fig. 1 illustrates a reconfigurable robot in three configurations: planar configuration with long links (A), planar configuration with short links (B), and anthropomorphic configuration $(\mathrm{C})$.

The kinematics model of a manipulator can be derived by the Screw notation; e.g. the Screw along xaxis by arm length and twist angle. This notion forms the basis for the mechanical design. Notably, a single telescopic joint with circular cross-section (as a connecting rod between two active joints) can deliver a translation motion along its axis, and rotation motion about the same axis. In order to simplify the design, the passive joints don't have any actuator or sensor. Also components of the locking device including the

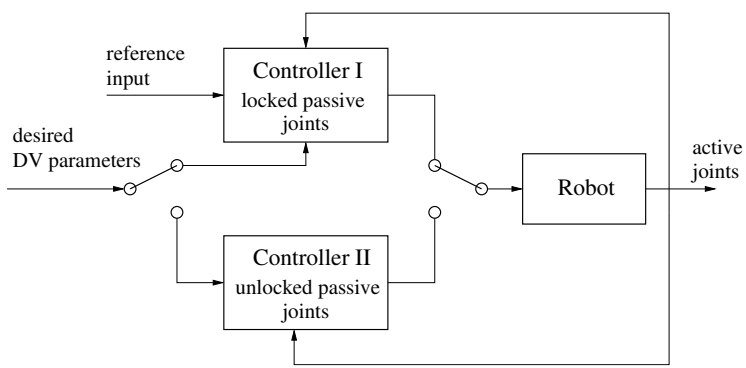

Figure 2: The control architecture.

solenoid can be installed inside the inner link resulting in a compact design.

The passive joints are controllable and observable when the the robots end-effector is constrained provided that the number of released passive joints is equal or less than the number of degrees-of-freedom lost due to the constraint, Fig. 3. Operation of the reconfigurable robot requires the robot control operates in two regimes as illustrated in Fig. 2. The robot control switches to Controller I when the brakes are locked, but it switches to controller II when the robot is constrained and the passive joint's brakes are released.

The process of changing manipulator configuration follows these sequences:

1. Determine optimal DV parameters from a given task specifications. The parameters constitutes the desired values of the passive joints.

2. Guide the robot towards a grasping point. After grasping is complete, release the brakes of the passive joints.

3. Switches to the constrained motion controller, i.e. controller II, to regulate the passive joints to their desired values.

4. Upon convergence of the joint error to zero, engage the brakes of all passive joints. Subsequently switches to Controller I after updating the tuning the controller according to the new robot parameters. 


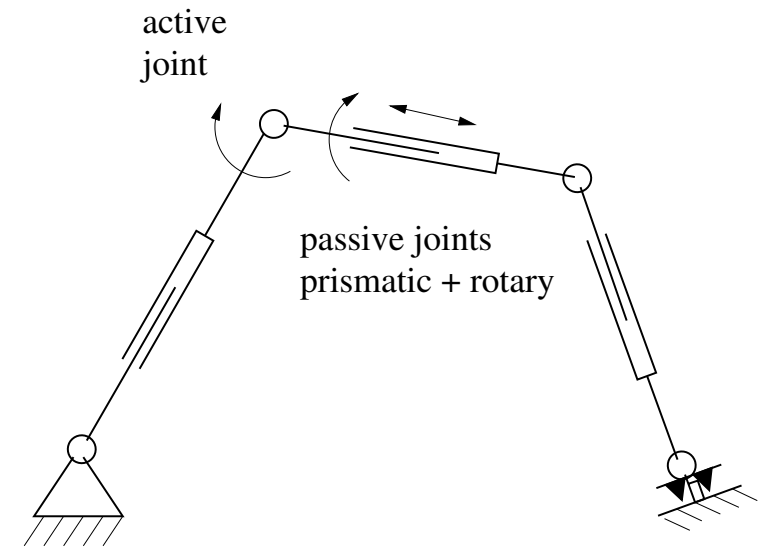

Figure 3: A reconfigurable robot.

\section{Kinematics of the Reconfig- urable Robots}

The transformation from the end-effector frame $\{N\}$ to frame $\{0\}$ is

$$
{ }_{0}^{n} T=\prod_{i=1}^{n}{ }_{i}^{i-1} T
$$

where each homogenous transformation can be written by the screw notation [13] as

$$
{ }_{i}^{i-1} T=\operatorname{Screw}_{x}\left(a_{i-1}, \alpha_{i-1}\right) \operatorname{Screw}_{z}\left(d_{i}, \theta_{i}\right),
$$

$\operatorname{Screw}_{x}\left(a_{i-1}, \alpha_{i-1}\right)=\left[\begin{array}{cccc}1 & 0 & 0 & a_{i-1} \\ 0 & c \alpha_{i-1} & -s \alpha_{i-1} & 0 \\ 0 & s \alpha_{i-1} & c \alpha_{i-1} & 0 \\ 0 & 0 & 0 & 1\end{array}\right]$.

In the above, $\theta_{i}, d_{i}, a_{i-1}$, and $\alpha_{i-1}$ are the DenavitHartenberg (DV) parameters corresponding to the joint angle, link offset, link length, and twist angle, respectively. Let $p_{i}=\left[a_{i}, \alpha_{i}\right]$ denote the screw parameters along $\mathrm{x}$, and $\theta=\left[\theta_{1}, \cdots, \theta_{n}\right]^{T} \in \mathbb{R}^{n}$ be joint variables. We assume that $p^{T}=\left[p_{1}^{T}, \cdots, p_{m}^{T}\right] \in \mathbb{R}^{2 m}$ are adjustable parameters, and $d_{i}$ are fixed parameters. Then, the transformation is written as function of $\theta$ and $p$, i.e. ${ }_{0}^{n} T={ }_{0}^{n} T(\theta, p)$, and so is the endeffector pose

$$
x=x(p, \theta) .
$$

Where $x \in \mathbb{R}^{n}$ denotes the position and orientation of the end-effector. In the sequel, $\theta$ and $p$ are treated as the active joints and passive joints, respectively consider $a_{i}$ and $\alpha_{i}$ as prismatic and rotary joints. The active joints are equipped with actuator and sensor, but the passive joints have neither actuator nor sensor. Also the passive joints are locked when the robot operates in free-space.
Let $p_{0}$ denote the kinematics parameters when the passive joints are locked and they are assumed known. Unlocking the passive joints gives rise to the number of the robot's joint coordinates, that is vector $q^{T}=\left[\begin{array}{ll}\theta^{T} & p^{T}\end{array}\right]$ represents the augmented joint coordinates. Then for the constrained robot, we have

$$
\Phi(q)=x\left(\theta, p_{0}\right)-x(\theta, p)=0
$$

Consider $\theta$ and $p$ as known and unknown variables in the above equation - recall that the value of $\theta$ is available in real-time. Then, $p$ can be obtained uniquely if $2 m \leq n$ and the nonlinear equations are independent. Assume that there exists explicit function $\Psi(\cdot)$ relating the passive joints and active joints by

$$
p=\Psi(\theta)
$$

Then,

$$
\dot{q}=\Lambda \dot{\theta}, \text { where } \Lambda=\left[\begin{array}{c}
I \\
\frac{\partial \Psi}{\partial \theta}
\end{array}\right]
$$

That is the active joints and passive joints can be treated as the independent and dependent coordinates.

\section{Control}

Consider the passive joints and active joints as a whole. Let's assume that the dynamics and kinematics models of the robot is available as a functions of the entire joint coordinates, i.e. $M=M(\theta, p)$ and $J=J(\theta, p)$. Then, the parameters of the Controller I can be trivially updated as the values of the passive joints are fixed and known. The main challenge lies in development of Controller II as the system has some joints without actuator.

The control objective is to regulate the passive joints of the system Fig. 3 to desired values $p_{d}$. This is tantamount to control the constrained mechanical system where the desired values of the independent coordinate is

$$
\theta_{d}=\Psi^{-1}\left(p_{d}\right)
$$

The equations describing dynamics of the mechanical system containing constraint can be written as [14]

$$
\begin{gathered}
M \ddot{q}+h(q, \dot{q})=f-\mathcal{F}, \\
\Phi(q)=0 .
\end{gathered}
$$

where $f \in \mathbb{R}^{n+2 m}$ is vector of generalized force, $\mathcal{F}$ is the constraint force, $M$ is inertia matrix, $h$ is the nonlinear vector, and $A=\partial \Phi / \partial q$ is the Jacobian of the 
constraint with respect to the augmented coordinate. Let $P=1+A^{+} A$ represent the projection on to the null-space of the Jacobian, i.e. $P \in \mathcal{N}(A)$.

In the light of the projection definition, the generalized force can be decomposed into two orthogonal subspaces

$$
f=f_{\|}+f_{\perp}
$$

where $f_{\|} \in \mathcal{N}$ and $f_{\perp} \in \mathcal{N}^{\perp}$ - one can show that $f_{\perp}$ doesn't contribute to the motion of the system [15]. Since the constraint force lies in the tangential space, i.e. $\mathcal{F} \in \mathcal{R}\left(A^{T}\right)$, the projection operator $P$ is an annihilator for the constraint force, i.e. $P \mathcal{F}=0$. Therefore, the constraint force can be eliminated from equation (3) if the equation is projected onto $P$. By premultiplying both sides of the equation by $P$ we have

$$
P M \ddot{q}=P(f-h) .
$$

Now, consider the following control law

$$
f_{\|}=P(h+M u)
$$

where

$$
u=\left(\dot{\Lambda}-\Lambda G_{D}\right) \dot{\theta}+G_{P}\left(\theta_{d}-\theta\right),
$$

and $G_{D}>0$ and $G_{P}>0$ are feedback gains. One can show that the control law stabilizes exponentially the positioning error [15], i.e.

$$
P M \Lambda(\ddot{e}+\dot{e}+e)=0,
$$

where $e=\theta_{d}-\theta$, and $e \rightarrow 0$. Hence $\theta \rightarrow \theta_{d}$ as $t \rightarrow \infty$. However, since the passive joints cannot generate any force, the generalized force has to have this form

$$
\left.f=\left[\begin{array}{c}
f_{1} \\
\vdots \\
f_{n} \\
0 \\
\vdots \\
0
\end{array}\right]\right\} \text { passive joints }
$$

Therefore, an admissible generalized force should satisfy

$$
f \in \mathcal{R}(B), \quad \text { and } \quad B=\left[\begin{array}{ll}
I & 0 \\
0 & 0
\end{array}\right],
$$

Now, we need to modify the motion control law (5) so that the condition in (6) is fulfilled. To this end, we add a component in $\mathcal{N}^{\perp}$, say $f_{\perp}$, so that $f=f_{\|}+f_{\perp} \in$ $\mathcal{R}(B)$. Recall that that $f_{\perp}$ does not affect the system motion at all. Hence, the motion tracking performance

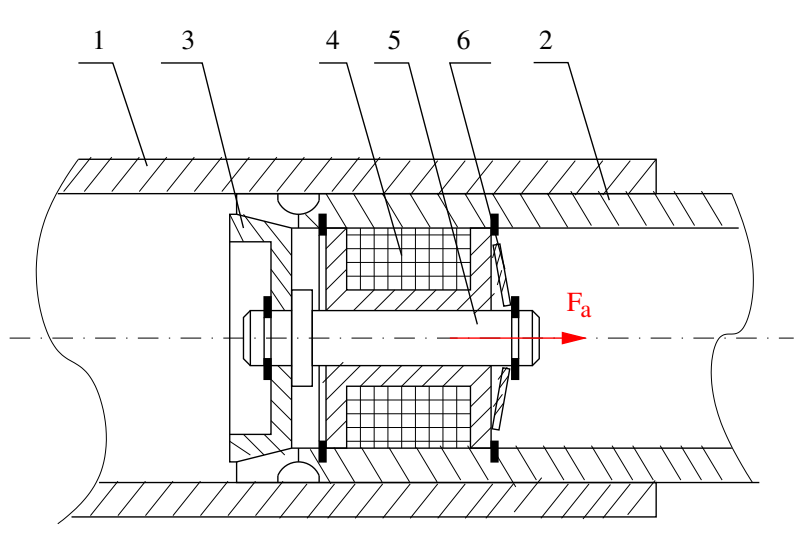

Figure 4: Design of the built-in brake; 1-outer hollow link, 2- inner hollow link, 3- tapered disc, 4- solenoid coil, 5-solenoid shaft 6 -disk spring.

is preserved by the enhancement. Let's assume $f_{\perp}=$ $(I-P) \eta$, where $\eta \in \mathbb{R}^{n}$. Then, we seek $\eta$ such that

$$
\begin{aligned}
f \in \mathcal{R}(B) & \Leftrightarrow(I-B) f=0 \\
& \Leftrightarrow Q \eta=-(I-B) f_{\|}
\end{aligned}
$$

where $Q=I-B-P+B P$, and $\eta$ is the unknown variable. Then, the particular solution can be found via pseudo-inversion, i.e.

$$
\eta=-Q^{+}(I-B) f_{\|}
$$

Hence $f_{\perp}=H f_{\|}$where $H=-(I-P) Q^{+}(I-B)$. Finally, we arrive at the motion control law for contained mechanical systems having passive joints

$$
f=(I+H) f_{\|},
$$

where $f_{\|}$is previously derived from (5).

\section{Design of Brake Mechanism}

Both linear and rotation motions of a passive joint can be easily achieved in a single telescopic link with circular cross section, see Fig. 4. Also a mechanism creating sufficient friction between the two slider links can simultaneously constrain both motions. The brake should be normally locked for minimum power consumption and for safety considerations. Also the brake should come with a compact design in order to be accommodated inside the link.

Fig. 4 illustrates the design of a brake mechanism. The collar at the end of the inner link is radially flexible and that allows development of a normal force, and hence friction, at the endpoint interface of the inner 


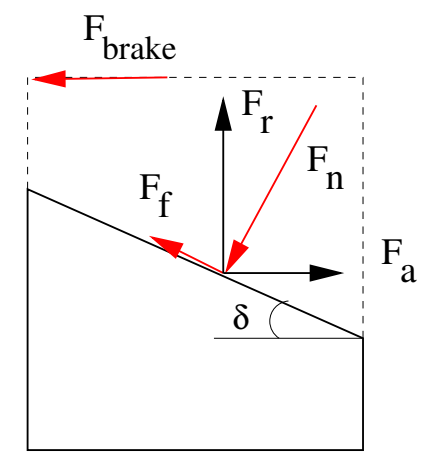

Figure 5: Force reactions on the tapered disc.

link and the outer link if the tapered disc is pushed in axially. The disc spring creates the axial force through the shaft and consequently the two slider links are normally locked. The solenoid can counterbalance the spring force if it is activated and thereby the brake is released.

The minimum axial force required to maintain the braking force is a decisive factor in the design of the brake because that determines the size of the solenoid. The following static analysis relates the maximum brake force to the axial force. Fig. 5 illustrates the force reactions on the tapered disc. $F_{n}$ and $F_{f}$ are the normal force and friction force which are in balance with the axial force $F_{a}$ and the radial force $F_{r}$. The radial force is transmitted through the flexible collar and hence it determines the maximum friction force which locks the two links together. The followings equations hold:

$$
\begin{aligned}
F_{a} & =F_{n} \sin \delta+F_{f} \cos \delta \\
F_{r} & =F_{n} \cos \delta-F_{f} \sin \delta \\
F_{f} & =\mu F_{n} \\
F_{\text {lock }} & =\mu F_{r}
\end{aligned}
$$

Substituting the first three equations in the last one yields

$$
F_{\text {lock }}=K F_{a}, \text { where } K=\mu \frac{1-\mu \tan \delta}{\tan \delta+\mu}
$$

is the mechanical advantages of the system. Note that large $K$ is desirable because then solenoid needs to produce less force. It is evident from (9) that $\delta=$ $\tan ^{-1} \mu \Rightarrow K=0$ and $\delta \approx 0 \Rightarrow K \approx 1$. Therefore, the tapered angle should be small as possible considering manufacturing limitation.

\section{Conclusion}

We have introduced the reconfigurable robots with passive joint which allows changing the robot's DV parameters. Unlike the conventional reconfigurable robots, this robot is not modular leading to a simple design. The kinematics analysis and control synthesis of such a reconfigurable robot have been presented. The design of a built-in brake for locking the passive joint has been also described.

\section{References}

[1] J. Angeles, Fundamentals of Robotic Mechanical Systems. Theory, Methods, and Algorithms. New York: Springer-Verlag, 1997.

[2] D. Schmitz, P. Khosla, and T. Kanade, "The CMU reconfigurable modular manipulator system," tech. rep., Carnegie Mellon University, Pittsburgh, PA, 1988.

[3] T. Fukuta and Y. Kawauchi, "Cellular robotic system (CEBOT) as one of the realization of selforganizing intelligent univeral manipulator," in Proce. IEEE Int. Conf. Robotics and Automation, pp. 662-667, 1990.

[4] C. Paredis and P. Khosla, "Design of modular fault tolerant manipulators," in Proc. First Workshop Algorithmic Foundations of Robotics, pp. 371-383, 1995.

[5] E. Yoshida, S. Murata, S. Kokaji, K. Tomita, and H. Kurokawa, "Micro self-reconfigurable robotic system using shape memory alloy," in Proc. Distributed Auton. Robotic Systems, pp. 145-155, 2000 .

[6] Murata, H. Kurokawa, and S. Kokaji, "Selfassembling machine," in Proc. IEEE Int. Conf. Robotics Automation, pp. 441-448, 1994.

[7] K. Kotay, D. Rus, M. Vona, and C. McGray, "The self-reconfiguring robot molecule," in Proc. IEEE Int. Conf. Robotics Automation, pp. 424431, 1998.

[8] D. Rus and M. Vona, "A basis for selreconfiguring robots using crystal modules," in Proc. IEEE/RSJ Int. Conf. Intell. Robots Systems, pp. 2194-2202, 2000. 
[9] S. Farritor, S. Dubowsky, N. Rutman, and J. Cole, "A systems level modular design approach to field robotics," in Proc. IEEE Int. Conf. Robotics Automation, pp. 2890-2895, 1996.

[10] A. Castano, A. Behar, and P. Will, "The conro modules for reconfigurable robots," IEEE Trans. on Mechatonics, pp. 100-106, 2002.

[11] G. Hamlin and A. C. Sanderson, Tetrobot: A Modular Approach to Reconfigurable Parallel Robotics. Boston, MA: Kluwer Publishers, 1998.

[12] B. Khoshnevis, P. Will, and W. M. Shen, "Highly compliant and self-tightening docking modules for precise and fast connection of self-reconfigurable robots," in IEEE Int. Conf. Robotics Automation, pp. 2311-2316, 2003.

[13] J. J. Craig, Introduction to Robotics: Mechanical and Control. Reading, Massachusetts: AddisonWesley Publishing Company, 2nd ed., 1989.

[14] J. G. de Jalon and E. Bayo, Kinematic and Dynamic Simulation of Multibody Systems. Springer-Verlag, 1989.

[15] F. Aghili, "Inverse and direct dynamics of constrained multibody systems based on orthogonal decopmosition of generalized force," in Proceedings of the IEEE Conference on Robotics and Automation, (Taipei, Taiwan), pp. 4035-4041, 2003. 